\title{
L'ostéoprotégérine et les douleurs osseuses d'origine cancéreuse
}

Chez les patients atteints de cancers primitifs des os ou de métastases osseuses, les douleurs osseuses sont très fréquentes et de traitement souvent difficile. D'abord sourdes et continues, elles s'aggravent avec l'évolution de la maladie et s'accompagnent d'épisodes douloureux aigus soit spontanés soit provoqués par les mouvements ou la pression. Si l'on sait que, dans la plupart des cas, l'intensité de ces douleurs reflète l'importance de la destruction osseuse, les facteurs qui contribuent à leur apparition, à leur maintien et à leur aggravation sont en revanche encore peu connus. L'os minéralisé et la moelle osseuse sont innervés par des fibres sensorielles afférentes, mais celles-ci sont très clairsemées en comparaison de leur abondance dans le périoste. De même, l'innervation de la tumeur est peu développée. Au fur et à mesure que l'ostéolyse s'accentue, les ostéoclastes (qui assurent normalement la résorption osseuse) et les cellules tumorales établissent un contact de plus en plus étroit avec les fibres nerveuses afférentes de l'os et du périoste, ce qui pourrait être directement à l'origine de ces douleurs. Les ostéoclastes créent en effet, au site de résorption osseuse, un environnement extracellulaire acide [1], ce qui pourrait stimuler les fibres sensorielles qui expriment des canaux ioniques sensibles au pH [2]. Par ailleurs, les cellules tumorales ou les macrophages présents au niveau des tumeurs libèrent de nombreux facteurs tels l'IL-1 (interleukin-1) ou le TNF- $\alpha$ (tumor necrosis factor $\alpha$ ) susceptibles, eux aussi, de sensibiliser ou de stimuler ces fibres sensorielles [3].

Schwei et al. ont décrit récemment un modèle murin de cancer osseux obtenu par l'injection de cellules sarcomateuses (lignée 2472) dans le canal médullaire du fémur [4]. On observe, 21 jours après l'injection, une destruction osseuse massive ainsi qu'une invasion du périoste par la tumeur. Les tests comportementaux indiquent clairement l'existence de douleurs aussi bien spontanées que provoquées par la palpation du membre, l'intensité de ces douleurs étant corrélée au degré de destruction osseuse. Il existe une sensibilisation des fibres nerveuses sensorielles afférentes et une réorganisation neurochimique au niveau de la corne dorsale de la moelle épinière, qui contient les neurones spinaux de la nociception et les interneurones impliqués dans les systèmes de contrôle segmentaire spinaux de la douleur ( $m / s$ 1998, $n^{\circ} 6-7$, p. 805). Cette réorganisation est probablement le témoin d'une sensibilisation centrale. Elle est localisée exclusivement du côté homolatéral à la lésion osseuse, entre les vertèbres lombaires L2 et L5, et est semblable à ce que l'on observe au cours des douleurs chroniques: hypertrophie des astrocytes sans perte neuronale, et expression, par les neurones des couches III à $\mathrm{V}$, de la protéine c-Fos $(\mathrm{m} / \mathrm{s} 1999$, $\left.n^{\circ} 10, p .1126\right)$ et de la dynorphine. Toutes ces modifications neurochimiques participent probablement à la persistence et à l'aggravation des douleurs osseuses et sont, là encore, corrélées à l'intensité de la destruction osseuse.

Ces observations ont conduit la même équipe à formuler l'hypothèse selon laquelle inhiber l'activité des ostéoclastes pourrait être un des moyens d'agir sur les douleurs osseuses d'origine cancéreuse. Cette inhibition devient possible grâce à la découverte du facteur de différenciation des ostéoclastes, l'OPGL (osteoprotegerin ligand), et de ses récepteurs. L'OPGL stimule la différenciation des précurseurs ostéoclastiques en se fixant sur son récepteur RANK, un membre de la famille des récepteurs du TNF (m/s 1999, $n^{\circ} 8-9$, p. 990). Ce facteur est synthétisé notamment par les cellules stromales, les ostéoblastes, les lymphocytes T activés $(\mathrm{m} / \mathrm{s} 2000$, $n^{\circ} 2, p .278$ ), et les cellules géantes de certains cancers primitifs ostéolytiques des os [5,6], et pourrait être en partie impliqué dans la destruction osseuse provoquée par les cancers (figure 1). Il existe un autre récepteur de l'OPGL, l'OPG (osteoprotegerin), soluble celui-ci, et qui entre en compétition avec RANK pour la fixation de l'OPGL, le détournant ainsi de son action sur la différenciation des ostéoclastes. L'OPG devenait ainsi logiquement un élément clé capable d'inhiber la résorption osseuse d'origine cancéreuse, ce que démontrent aujourd'hui Honore et $a l$. dans leur modèle murin de métastases osseuses [7]. L'injection quotidienne d'OPG, débutée cinq jours après celle des cellules sarcomateuses dans le fémur, réduit en effet le nombre d'ostéoclastes et prévient la destruction osseuse induite par la tumeur. De façon remarquable, ce traitement entraîne une sédation des douleurs osseuses ainsi que de tous les signes caractéristiques de la sensibilisation nerveuse centrale et périphérique. Il est donc clair que cette sensibilisation ne pouvait être attribuée à un effet direct des cellules tumorales sur les fibres sensorielles puisque le traitement par l'OPG n'a aucun effet sur le volume de la tumeur, et que l'injection des cellules tumorales, non plus dans le fémur, mais dans le quadriceps, ne provoque aucune douleur [4]. Enfin, l'administration d'OPG est nettement plus efficace sur les douleurs provoquées que sur celles qui apparaissent spontanément. Cela suggère 


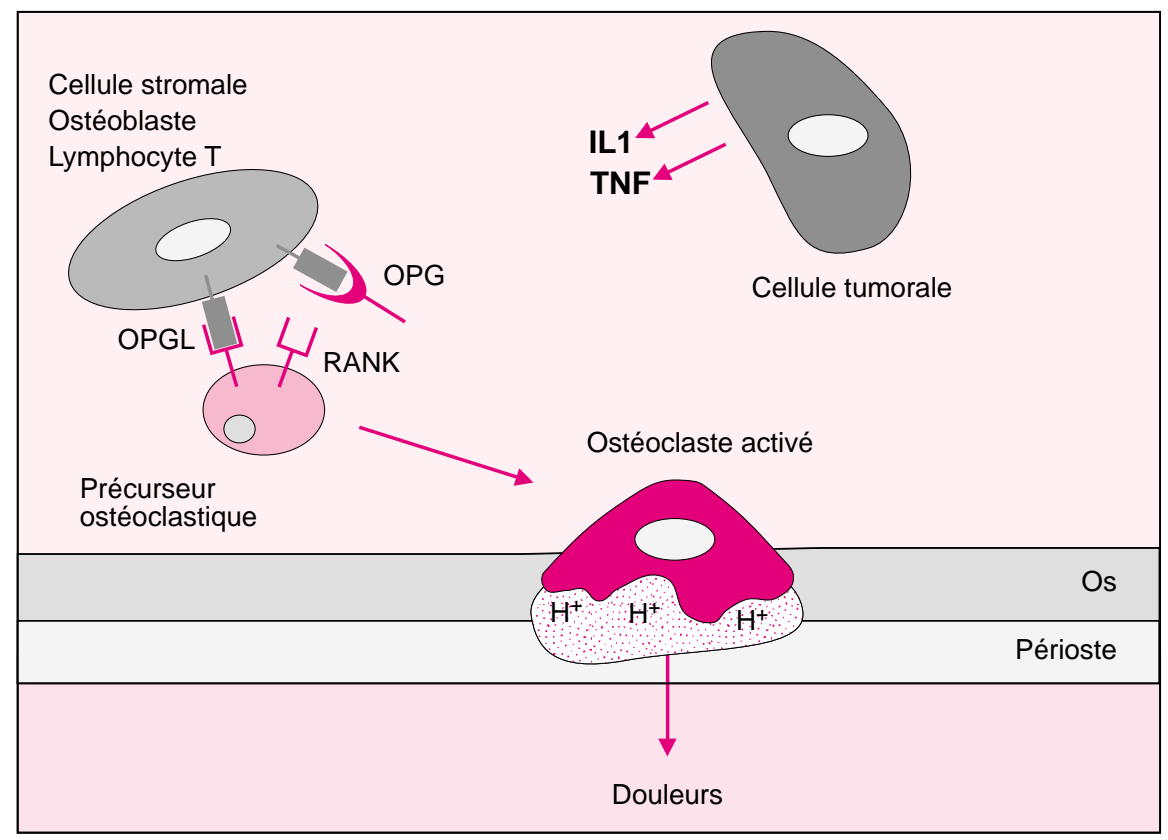

Figure 1. Mécanismes possibles des douleurs osseuses d'origine cancéreuse. Les cellules tumorales sécrètent des cytokines susceptibles d'induire la synthèse d'OPGL (osteoprotegerin ligand) par les cellules stromales, les ostéoblastes ou les lymphocytes $T$ activés. L'OPGL, en se fixant sur son récepteur RANK à la surface des précurseurs ostéoclastiques, induit leur différenciation en ostéoclastes matures qui sont à l'origine de la résorption osseuse. La diminution du $\mathrm{pH}$ extracellulaire au niveau du site de résorption osseuse ainsi que les facteurs sécrétés par les cellules tumorales pourraient activer et sensibiliser les fibres nerveuses nociceptives afférentes qui innervent le périoste. L'administration d'OPG (osteoprotegerin), en bloquant I'interaction de l'OPGL avec son récepteur RANK, empêche la maturation et l'activation des ostéoclastes et inhibe la destruction osseuse, limitant ainsi l'apparition et l'aggravation des douleurs.

que l'activité des ostéoclastes est plutôt responsable des douleurs provoquées tandis que d'autres facteurs, probablement sécrétés par les cellules tumorales, pourraient être à l'origine des douleurs sur celles qui apparaissent spontanément.

Ces résultats apportent de nouveaux éléments pour la compréhension des mécanismes responsables des douleurs osseuses au cours des destructions osseuses d'origine cancéreuse et sur le rôle très probable de l'OPGL et de ses récepteurs dans le développement de ces lésions (figure 1). Il reste toutefois à détermi-

' lement efficace à un stade plus tardif de la maladie, quand la destruction osseuse est déjà importante, ce qui correspond le plus souvent au stade auquel les métastases osseuses sont découvertes. Les résultats obtenus dans un modèle murin d'hypercalcémie maligne d'origine tumorale, montrant que l'injection d'OPG est non seulement capable de prévenir la lyse osseuse et l'hypercalcémie, mais aussi de les traiter après leur apparition [8], sont très encourageants. Chez l'homme, avant toute application thérapeutique, il faudra confirmer que la destruction osseuse au cours des cancers procède des mêmes mécanismes. Enfin, les premiers résultats des essais cliniques de phase I et II concernant l'emploi de l'OPG dans l'ostéoporose postménopausique devraient bientôt préciser si ce traitement provoque des effets indésirables susceptibles de limiter ou d'interdire son utilisation.

1. Delaisse JM, Vaes G. Biology and physiology of the osteoclast. In: Rflain BR, Gau CV, eds. Ann Harbor: CRC Press, 1992 : 289-314.

2. Olson TH, Riedl MS, Vulchanova L, Ortiz-Gonzalez XR, Elde R. An acid sensing ion channel (ASIC) localizes to small primary afferent neurons in rats. NeuroReport 1998; $9: 1109-13$.

3. Woolf CJ, Allchorne A, Safieh-Garabedian B, Poole S. Cytokines, nerve growth factor and inflammatory hyperalgesia: the contribution of tumor necrosis factor alpha. Br J Pharmacol 1997; 121: 417-24.

4. Schwei MJ, Honore P, Rogers SD, et al. Neurochemical and cellular reorganization of the spinal cord in a murine model of bone cancer pain. $J$ Neurosci 1999; 19: 10886-97.

5. Huang L, Xu J, Wood DJ, Zheng MH. Gene expression of osteoprotegerin ligand, osteoprotegerin, and receptor activator of $\mathrm{NF}-\mathrm{\kappa B}$ in giant cell tumor of bone: possible involvement intumor cell-induced osteoclast-like cell formation. Am J Pathol 2000; 156 : 761-7.

6. Atkins GJ, Haynes DR, Graves SE, et al. Expression of osteoclast differentiation signals by stromal elements of giant cell tumors.J Bone Miner Res $2000 ; 15: 640-9$.

7. Honore P, Luger NM, Sabino MA, et al. Osteoprotegerin blocks bone cancer-induced skeletal destruction, skeletal pain and pain-related neurochemical reorganization of the spinal cord. Nat Med 2000; 6: 521-8.

8. Capparelli C, Kostenuik PJ, Morony S, et al. Osteoprotegerin prevents and reverses hypercalcemia in a murine model of humoral hypercalcemia of malignancy. Cancer Res $2000 ; 15$ : 783-7.

\section{Remerciements}

Je remercie Pierre Marie et Bernard Calvino pour la relecture de ce manuscrit.

\section{Pascale Borensztein}

Inserm U.474, Maternité Port-Royal, 123, boulevard de Port-Royal, 75014 Paris, France. 